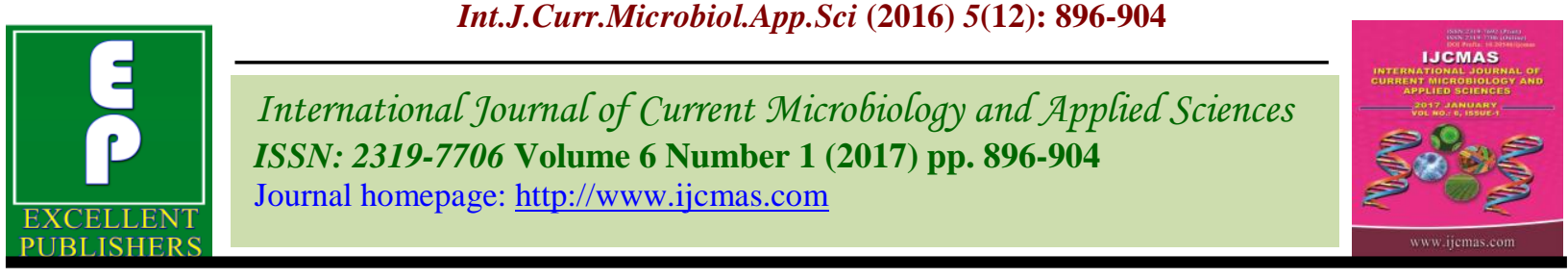

Original Research Article

http://dx.doi.org/10.20546/ijcmas.2017.601.105

\title{
Screening of Chickpea Varieties, Cultivars and Genotypes against Fusarium oxysporum f. sp. ciceri
}

\author{
D.S. Thaware ${ }^{1}$, V.M. Gholve ${ }^{2}$ and P.H. Ghante ${ }^{2}$ \\ ${ }^{1}$ Department of Plant Pathology, Ratnai College of Agriculture, Akluj (MPKV., Rahuri) \\ 431 101, (Maharashtra), India \\ ${ }^{2}$ Department of Plant Pathology, Marathwada College of Agriculture, Parbhani \\ (VNMKV, 431401 Parbhani), (Maharashtra), India
}

*Corresponding author

A B S T R A C T

\begin{abstract}
Keywords
Cicer arietinum,

Fusarium wilt, genotypes, cultivars and resistance.

Article Info

Accepted:

28 December 2016

Available Online:

10 January 2017
\end{abstract}

Fusarium oxysporum $\mathrm{f}$. sp. ciceri is one of the most destructive pathogen, causing wilt disease in chickpea and thereby inflicting accountable quantitative as well as qualitative losses. Under natural epiphytotic conditions (Rabi 2013-2014), all the 50 chickpea entries exhibited different reactions against Fusarium oxysporum f. sp. ciceri. However, six test entries were found highly resistant (Vishal, BCP-10, BCP-21, BCP-49, BCP-60 and BCP-61), thirty one were resistant, eight were moderately resistant (BDNG 9-3, BDNG-2003-1, JAKI- 9218, BDNG-2010-1, BDNG- 801, AKG-12009, PKV Kabuli-2 and BCPK-3), two were moderately susceptible (PKV Kabuli-4 and Virat) and three were highly susceptible (JG-62, BDNGK-807 and AKG-1207). In the Rabi 2014-2015, a total 48 entries exhibited different reactions against Fusarium wilt of chickpea. Single test entry was found highly resistant (PG8108 ), twenty one were resistant, eight were moderately resistant, ten were moderately susceptible (AKG-1306, BDNG-801, Jaki-9218, BDNG K-799, PKV Kabuli-4, BDNG-798, Krupa, PG-12310, PG-12138 and PG-0611-14), five were susceptible (AKG-1103, B.D.N. 9-3, BDNG-K-807, AKG-1208 K and PKV Kabuli-2) and three were highly susceptible (JG-62, BDNG-804 and BDNG-2013-2).

\section{Introduction}

Chickpea (Cicer arietinum L.) is an important pulse crop, which belongs to leguminoceae family, ranking third after dry beans (Phaseolus vulgaris L.) and dry peas (Pisum sativum L.) (Dhar and Gurha, 1998). The centre of origin is in Eastern Mediterranean. India is largest producer of chickpea in world sharing 65.25 per cent in area and 65.49 per cent in production.
In India chickpea is grown on 81.17 lakh ha area with production of 59.01 lakh tonnes and productivity of $727 \mathrm{~kg} / \mathrm{ha}$. The production of chickpea in Maharashtra is 7.54 lakh tonnes with productivity of 574 $\mathrm{kg} / \mathrm{ha}$ which covered nearly 13.15 lakh ha of area. Maharashtra contributes about 14 per cent share in total production of country (Anonymous, 2015). Chickpea is cultivated 
throughout the state Maharashtra and widely grown in regions of Western Maharashtra, Marathwada and Vidhrabha.

The major limiting factor in chickpea production is Fusarium wilt which is caused by $F$. oxysporum Schlechtend. Fr. f. sp. ciceris (Padwick) Matuo and K. Sato. (Nene and Reddy, 1987). It was first reported in Indo-Pak sub-continent (Butler, 1918). At national level the yield losses encountered due to wilt may vary between 5 to 10 per cent (Singh and Dahiya, 1973).

It observed damage to be upto 61 per cent at seedling stage and 43 per cent at flowering stage (Nema and Khare, 1973). In general, the disease causes substantial yield losses which may reach even 100 per cent under favourable weather conditions (Jalali and Chand, 1992). The chickpea is cultivated as a rain fed crop in Maharashtra state and yield losses amounted to 10 to 15 per cent (Khillare et al., 2009)

\section{Materials and Methods}

Screening of chickpea varieties, cultivars and genotypes

The experiment was conducted in wilt sick plot, Agricultural Research Station Badnapur, VNMKV, Parbhani (M.S.) under field condition, to see the response of different chickpea varieties, cultivars and genotypes against Fusarium oxysporum f. sp. ciceri. Surface sterilized $\left(0.1 \% \mathrm{HgCl}_{2}\right)$ seeds of all test entries of chickpea were sown (15 October) in wilt sick soil (Fusarium oxysporum f. sp. ciceri). All test entries alternated with susceptible check JG62 were planted in two replications and the length of each row was 5 meter. The observations viz., total number of plants, total number of infected plants and the average of wilt plants in each entry were calculated. The observations on wilt were recorded in percentage of disease incidence was recorded by applying 0-9 point disease rating scale (IIPR, 1999).

\section{Grade for accounting per cent mortality of Fusarium oxysporum f. sp. Ciceri}

\begin{tabular}{ll}
\hline Grade & Per cent mortality \\
0 & No disease \\
1 & 1 to 10 \\
2 & 10.1 to 20 \\
3 & 20.1 to 30 \\
4 & 30.1 to 50 \\
5 & 50 and above \\
\hline
\end{tabular}

\section{Results and Discussion}

\section{Rabi 2013-2014}

In order to find out the sources of resistance in chickpea for Fusarium wilt, fifty and forty eight chickpea genotypes which were collected from Agricultural Research Station, VNMKV, Parbhani were evaluated

\author{
Disease reactions \\ Highly resistant (HR) \\ Resistant (R) \\ Moderately resistant (MR) \\ Moderately susceptible (MS) \\ Susceptible (S) \\ Highly susceptible (HS) \\ during Rabi 2013-14 and 2014-15
}

respectively, both under field conditions by the standard procedure. Results (Table 1) revealed that, during Rabi 2013-2014 at under natural epiphytotics, all the 50 chickpea entries exhibited different reactions against Fusarium oxysporum f. sp. ciceri. However, six cultivars viz., Vishal, BCP-10, BCP-21, BCP-49, BCP-60 and BCP-61 
were found highly resistant with mean disease intensity of 00.00 per cent, thirty one varieties, cultivars, germplasm lines viz., BDNG-2013-1, BCP-26, SAKI- 9516, AKG-1109, AKG-1209, AKG-1108, Vijay, PG-0752, PG-8108, PG-302-10, Digvijay, AKG-70, AKG-1009, JAKI-1218, AKG20021K， BCPK-1， BCPK-2， BCPK-2, Krupa, BCP-92, BCP-51, BCP-24, BCP-52, BCP-124, BCP-37, BCP-11, BCP-36, BCP48, BCP-60, BCP-26 and BCP-28 were found moderately resistant with mean disease intensity in the range of 1.25 (BCP37) to 9.55 (BDNG-797 and BCPK-2) per cent, eight varieties, cultivars, germplasm lines viz., BDNG-9-3, BDNG-2003-1, JAKI9218, BDNG-2010-1, BDNG-801, AKG12009, PKV Kabuli-2 and BCPK-3 were found moderately resistant with mean disease intensity in the range of 12.69 (PKV Kabuli-2) to 22.25 (AKG-12009) per cent. Two varieties PKV Kabuli-4 and Virat was found moderately susceptible with mean disease intensity of 32.40 and 29.16 per cent; whereas, JG-62 (100\%), BDNGK-807 $(100 \%)$ and AKG-1207 (89.18\%) were found highly susceptible to wilt.

The observations on per cent wilt incidence were recorded at 15 days before harvesting of the crop and test entries of chickpea were graded and categorized as highly resistant (00.00\%), resistant (1-10\%), moderately resistant (11-25\%) moderately susceptible (26-50\%), susceptible (51-75\%) and highly susceptible (76-100\%).

\section{Rabi 2014-2015}

Results (Table 3), revealed that, during Rabi 2014-2015 at under natural epiphytotics, all the 48 chickpea entries exhibited different reactions against Fusarium oxysporum f. sp. ciceri.

Table.1 Reaction of chickpea lines to wilt during Rabi 2013-2014

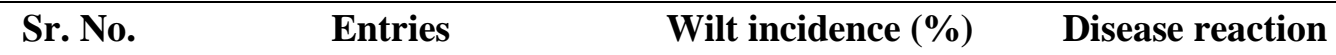

\section{Reaction of SMVT (R) chickpea lines to wilt disease}

$\begin{array}{llll}1 & \text { BDNG-2013-1 } & 02.57 & \text { Resistant } \\ 2 & \text { Krupa } & 01.72 & \text { Resistant } \\ 3 & \text { BDNG-9-3 } & 19.09 & \text { Moderately resistant } \\ 4 & \text { BCP-26 } & 01.92 & \text { Resistant } \\ 5 & \text { BDNG-2003-1 } & 14.58 & \text { Moderately resistant } \\ 6 & \text { SAKI- 9516 } & 05.31 & \text { Resistant } \\ 7 & \text { AKG-1109 } & 01.78 & \text { Resistant } \\ 8 & \text { AKG-1209 } & 07.78 & \text { Resistant } \\ 9 & \text { JAKI- 9218 } & 16.04 & \text { Moderately resistant } \\ 10 & \text { AKG-1108 } & 03.92 & \text { Resistant } \\ 11 & \text { Vijay } & 07.14 & \text { Resistant } \\ 12 & \text { PG-0752 } & 05.69 & \text { Resistant } \\ 13 & \text { Vishal } & 00.00 & \text { Highly resistant } \\ 14 & \text { PG-8108 } & 01.65 & \text { Resistant } \\ 15 & \text { PG-302-10 } & 02.63 & \text { Resistant } \\ 16 & \text { Digvijay } & 05.92 & \text { Resistant } \\ 17 & \text { JG-62 } & 100.00 & \text { Highly susceptible }\end{array}$




\section{Reaction of SMVT (I) chickpea lines to wilt disease}

$\begin{array}{lll}18 & \text { BCP-49 } & 00.00 \\ 19 & \text { BDNG-2010-1 } & 16.34 \\ 20 & \text { BDNG-801 } & 18.22 \\ 21 & \text { AKG-70 } & 01.78 \\ 22 & \text { AKG-2009 } & 22.25 \\ 23 & \text { AKG-1009 } & 05.61 \\ 24 & \text { JAKI-1218 } & 07.90 \\ 25 & \text { BCP-60 } & 00.00\end{array}$

\section{Reaction of SMVT (Kabuli) chickpea lines to wilt disease}

$\begin{array}{llc}26 & \text { AKG-1207 } & 89.18 \\ 27 & \text { PKV Kabuli-2 } & 12.69 \\ 28 & \text { AKG-20021 K } & 06.56 \\ 29 & \text { PKV Kabuli-4 } & 32.40 \\ 30 & \text { BCPK-1 } & 02.27 \\ 31 & \text { BCPK-2 } & 09.55 \\ 32 & \text { AKG-1205 } & 04.95 \\ 33 & \text { Virat } & 29.16 \\ 34 & \text { BCPK-3 } & 13.28 \\ 35 & \text { BDNGK-807 } & 100.00\end{array}$

\section{Reaction of BCP chickpea lines to wilt disease}

$\begin{array}{llll}36 & \text { BCP-92 } & 01.92 & \text { Resistant } \\ 37 & \text { BCP-51 } & 02.85 & \text { Resistant } \\ 38 & \text { BCP-24 } & 03.22 & \text { Resistant } \\ 39 & \text { BCP-52 } & 03.43 & \text { Resistant } \\ 40 & \text { BCP-124 } & 04.55 & \text { Resistant } \\ 41 & \text { BCP-21 } & 00.00 & \text { Highly resistant } \\ 42 & \text { BCP-37 } & 01.25 & \text { Resistant } \\ 43 & \text { BCP-61 } & 00.00 & \text { Highly resistant } \\ 44 & \text { BCP-10 } & 00.00 & \text { Highly resistant } \\ 45 & \text { BCP-11 } & 01.42 & \text { Resistant } \\ 46 & \text { BCP-36 } & 03.27 & \text { Resistant } \\ 47 & \text { BCP-48 } & 01.92 & \text { Resistant } \\ 48 & \text { BCP-60 } & 01.56 & \text { Resistant } \\ 49 & \text { BCP-26 } & 07.91 & \text { Resistant } \\ 50 & \text { BCP-28 } & 03.85 & \text { Resistant }\end{array}$

Highly resistant Moderately resistant Moderately resistant Resistant Moderately resistant Resistant

Resistant

Highly resistant

Highly susceptible

Moderately resistant

Resistant

Moderately susceptible

Resistant

Resistant

Resistant

Moderately susceptible

Moderately resistant

Highly susceptible 
Table.2 Categorization of chickpea cultivars / varieties, genotypes and germplasm lines

\begin{tabular}{|c|c|c|c|}
\hline Category & Reaction & $\begin{array}{r}\text { Average wilt } \\
\text { incidence (\%) }\end{array}$ & $\begin{array}{l}\text { Varieties / cultivars / genotypes } \\
\text { germplasm lines }\end{array}$ \\
\hline 0 & Highly resistant (6) & 00.00 & $\begin{array}{l}\text { Vishal, BCP-10, BCP-21, BCP-49, BCP-60 } \\
\text { and BCP-61 }\end{array}$ \\
\hline 1 & Resistant (31) & $1-10$ & $\begin{array}{l}\text { BDNG-2013-1, BCP-26, SAKI- 9516, AKG- } \\
\text { 1109, AKG-1209, AKG-1108, Vijay, PG-0752, } \\
\text { PG-8108, PG-302-10, Digvijay, AKG-70, } \\
\text { AKG-1009, JAKI-1218, AKG-20021 K, BCPK- } \\
\text { 1, BCPK-2, BCPK-2, Krupa, BCP-92, BCP-51, } \\
\text { BCP-24, BCP-52, BCP-124, BCP-37, BCP-11, } \\
\text { BCP-36, BCP-48, BCP-60, BCP-26 and BCP- } \\
28\end{array}$ \\
\hline 2 & $\begin{array}{l}\text { Moderately resistant } \\
\text { (8) }\end{array}$ & $11-25$ & $\begin{array}{l}\text { BDNG 9-3, BDNG-2003-1, JAKI- 9218, } \\
\text { BDNG-2010-1, BDNG- 801, AKG-12009, PKV } \\
\text { Kabuli-2 and BCPK-3 }\end{array}$ \\
\hline 3 & $\begin{array}{l}\text { Moderately } \\
\text { susceptible (2) }\end{array}$ & $26-50$ & PKV Kabuli-4 and Virat \\
\hline 4 & Susceptible (none) & $51-75$ & - \\
\hline 5 & Highly susceptible (3) & $76-100$ & JG-62, BDNGK-807 and AKG-1207 \\
\hline
\end{tabular}

Table.3 Reaction of chickpea lines to wilt during Rabi 2014-2015

\begin{tabular}{cccl}
\hline Sr. No. & \multicolumn{1}{c}{ Entries } & Wilt incidence (\%) & \multicolumn{1}{c}{ Disease reaction } \\
Reaction of SMVT $(\mathbf{R})$ chickpea lines to wilt disease & 12.49 & Moderately resistant \\
1 & P.G. $0302-10$ & 7.79 & Resistant \\
2 & AKG-1106 & 70.26 & Susceptible \\
3 & AKG-1103 & 8.94 & Resistant \\
4 & AKG-1108 & 5.82 & Resistant \\
5 & Saki- 9516 & 00.00 & Highly resistant \\
6 & PG-8108 & 3.88 & Resistant \\
7 & BCP-26 & 13.22 & Moderately resistant \\
8 & Vishal & 10.11 & Resistant \\
9 & PG-611-14 & 6.92 & Resistant \\
10 & BDNG-2013-1 & 93.14 & Highly susceptible \\
11 & BDNG-2013-2 & 3.57 & Resistant \\
12 & BDNG-2003-1 & 11.11 & Moderately resistant \\
13 & Jaki-9218 & 25.51 & Moderately resistant \\
14 & BDNG-797 & 60.85 & Susceptible \\
15 & BDN 9-3 & 100 & Highly susceptible \\
16 & JG-62 & & \\
Reaction of & SMVT (I) chickpea lines to wilt disease & Resistant \\
17 & PG-302-10 & 10.10 & Highly susceptible \\
18 & BDNG-804 & 95.83 & \\
\hline
\end{tabular}




\begin{tabular}{|c|c|c|c|}
\hline 19 & BDNG-2010-1 & 1.78 & Resistant \\
\hline 20 & BCP-60 & 3.50 & Resistant \\
\hline 21 & AKG-70 & 1.61 & Resistant \\
\hline 22 & AKG-1303 & 1.51 & Resistant \\
\hline 23 & AKG-1109 & 10.00 & Resistant \\
\hline 24 & AKG-1306 & 39.41 & Moderately susceptible \\
\hline 25 & AKG-1009 & 12.10 & Moderately resistant \\
\hline 26 & BDNG-801 & 37.24 & Moderately susceptible \\
\hline 27 & PG-8108 & 3.80 & Resistant \\
\hline 28 & Saki-9501 & 12.95 & Moderately resistant \\
\hline 29 & Jaki-9218 & 34.72 & Moderately susceptible \\
\hline 30 & ВСР-49 & 10.34 & Resistant \\
\hline 31 & Vishal & 5.77 & Resistant \\
\hline \multicolumn{4}{|c|}{ Reaction of SMVT (K) chickpea lines to wilt disease } \\
\hline 32 & BDNG K-807 & 55.03 & Susceptible \\
\hline 33 & BDNG K-799 & 43.95 & Moderately susceptible \\
\hline 34 & AKG-1208 K & 63.76 & Susceptible \\
\hline 35 & AKG-1304 K & 24.41 & Moderately resistant \\
\hline 36 & PKV Kabuli-4 & 45.97 & Moderately susceptible \\
\hline 37 & PKV Kabuli-2 & 56.45 & Susceptible \\
\hline 38 & BDNG-798 & 42.29 & Moderately susceptible \\
\hline 39 & Krupa & 30.35 & Moderately susceptible \\
\hline 40 & Virat & 10.83 & Resistant \\
\hline 41 & PG-12310 & 32.27 & Moderately susceptible \\
\hline 42 & PG-12138 & 41.21 & Moderately susceptible \\
\hline 43 & PG-12313 & 11.90 & Moderately resistant \\
\hline \multicolumn{4}{|c|}{ Reaction of SMVT (DR/DI/DL) chickpea lines to wilt disease } \\
\hline 44 & PG-0302-10 & 2.38 & Resistant \\
\hline $\begin{array}{l}45 \\
46 \\
47\end{array}$ & $\begin{array}{l}\text { PG-12110 } \\
\text { Digvijay } \\
\text { PG-0611-14 }\end{array}$ & $\begin{array}{c}2.17 \\
5.67 \\
43.33\end{array}$ & $\begin{array}{l}\text { Resistant } \\
\text { Resistant } \\
\text { Moderately susceptible }\end{array}$ \\
\hline 48 & PG-08108 & 2.17 & Resistant \\
\hline
\end{tabular}


Table.4 Categorization of chickpea varieties / cultivars, genotypes and germplasms lines

\begin{tabular}{|c|c|c|c|}
\hline Category & Reaction & $\begin{array}{l}\text { Average wilt } \\
\text { incidence (\%) }\end{array}$ & $\begin{array}{c}\text { Varieties / cultivars / genotypes } \\
\text { germplasms lines }\end{array}$ \\
\hline 0 & Highly resistant (1) & 00.00 & PG-8108 \\
\hline 1 & Resistant (21) & $1-10$ & $\begin{array}{l}\text { AKG-1106, AKG-1108, Saki- 9516, BCP- } \\
\text { 26, PG-611-14, BDNG-2013-1, BDNG- } \\
\text { 2003-1, PG-302-10, BDNG-2010-1, BCP- } \\
\text { 60, AKG-70, AKG-1303, AKG-1109, PG- } \\
\text { 8108, BCP-49, Vishal, Virat, PG-0302-10, } \\
\text { PG-12110, Digvijay and PG-08108 }\end{array}$ \\
\hline 2 & Moderately resistant (8) & $11-25$ & $\begin{array}{l}\text { P.G. 0302-10, Vishal, Jaki-9218, BDNG- } \\
\text { 797, AKG-1009, Saki-9501, AKG-1304 K } \\
\text { and PG-12313 }\end{array}$ \\
\hline 3 & Moderately susceptible (10) & $26-50$ & $\begin{array}{l}\text { AKG-1306, BDNG-801, Jaki-9218, } \\
\text { BDNG K-799, PKV Kabuli-4, BDNG- } \\
\text { 798, Krupa, PG-12310, PG-12138 and } \\
\text { PG-0611-14 }\end{array}$ \\
\hline 4 & Susceptible (5) & $51-75$ & $\begin{array}{l}\text { AKG-1103, B.D.N. 9-3, BDNG-K-807, } \\
\text { AKG-1208 K and PKV Kabuli-2 }\end{array}$ \\
\hline 5 & Highly susceptible (3) & $76-100$ & JG-62, BDNG-804 and BDNG-2013-2 \\
\hline
\end{tabular}

However, one cultivar PG-8108 was found highly resistant with mean disease intensity of 00.00 per cent, twenty one varieties, cultivars, germplasm lines viz., AKG-1106, AKG-1108, Saki-9516, BCP-26, PG-611-14, BDNG-2013-1, BDNG-2003-1, PG-302-10, BDNG-2010-1, BCP-60, AKG-70, AKG1303, AKG-1109, PG-8108, BCP-49, Vishal, Virat, PG-0302-10, PG-12110, Digvijay and PG-08108 were found moderately resistant with mean disease intensity in the range of 1.51 (AKG-1303) to 10.34 (BCP-49) per cent, eight varieties, cultivars, germplasms lines viz., P.G. 030210, Vishal, Jaki-9218, BDNG-797, AKG1009, Saki-9501, AKG-1304 K and PG12313 were found moderately resistant with mean disease intensity in the range of 11.11 (Jaki-9218) to 25.51 (BDNG-797) per cent. Ten varieties, cultivars, germplasm lines viz., AKG-1306, BDNG-801, Jaki-9218, BDNG-K-799, PKV Kabuli-4, BDNG-798, Kripa, PG-12310, PG-12138 and PG-0611-
14 were found moderately susceptible with mean disease intensity of 30.35 (Jaki-9218) and 45.97 (PKV Kabuli-4) per cent; whereas, AKG-1103, B.D.N. 9-3, BDNG-K807, AKG-1208 K and PKV Kabuli-2 were found susceptible with mean disease intensity of 70.26 (AKG-1103) and 56.45 (PKV Kabuli-2) per cent. Three varieties / cultivars, germplasm lines viz., JG-62 (100\%), BDNG-804 (95.83\%) and BDNG2013-2 (93.14\%), were found highly susceptible to wilt.

The disease free lines, resistant lines and moderately resistant lines can be utilized in resistant breeding programme towards incorporation of resistant genes in releasing varieties / hybrids. These results of the chickpea germplasms lines, varieties, cultivars reactions against Fusarium oxysporum f. sp. ciceri both under natural epiphytotics are on the same line as to that of reported earlier by several workers. 
Pawar et al., (1993), Zote et al., (1993), Suryawanshi et al., (2003), Chavan, (2004), Honnareddy and Dubey, (2006), Kohire et al., (2006), Trivedi and Gurha (2007), Mulik (2009), Ahmed (2010), Patil (2010), Korde, (2011), Mandhare et al., (2011) and Kumar et al., (2012) gave been screened number of chickpea genotypes and identified promising cultivars in Maharashtra and India.

\section{Acknowledgements}

The authors are thankful to Head, Agricultural Research Station Badnapur, VNMKV, Parbhani (M.S.) for funding and providing the valuable suggestions during our research period.

\section{References}

Ahmad M A 2010. Variability in Fusarium oxysporum f. sp. ciceri for chickpea wilt resistance in Pakistan. Ph. D. (Agri.) thesis, (Abs.) submitted to Quaid-i-Azam University, Islamabad, Pakistan.

Anonymous 2015. Directorate of Economics and Statistics, Department of Agriculture and Cooperation. Agricultural statistics at a glance. 90$96 \mathrm{pp}$.

Butler E J 1918. Fungi and diseases of plants. Book published. (M. C. Saxena, K. B. Singh, edi.), CABI Publishing, CAB Int. Wallingford, UK. 233-270 pp.

Chavan T B 2004. Studies on Fusarium oxysporum f. sp. ciceri (Padwick) Snyder and Hansen causing wilt of chickpea (Cicer arietinum L.). M. Sc. (Agri.) thesis submitted to Indira Gandhi Agriculture University, Raipur (India).

Dhar V and Gurha S N 1998. Integrated management of chickpea diseases. (Rajeev, K., Upadhyay, K. G.,
Mukerji, B. P., Chamola and Dubey, O. P. (edi.)), APH Pub. Co., New Delhi. (India). 249 pp.

Honnareddy N and Dubey S C 2006. Pathogenic and molecular characterization of Indian isolates of Fusarium oxysporum f. sp. ciceris causing chickpea wilt. Current Sci. 91 (5): 661-666.

IIPR 1999. Proceedings of technical programme for Rabi workshop organization by Indian Institute of Pulse Research Kanpur. 13 pp.

Jalali B L and Chand H 1992. Diseases of Cereals and Pulses. (U. S. Singh, A. N. Mukhopadhayay, J. Kumar, and H. S. Chaube, edi.) Prentice Hall, Englewood Cliffs, NY. 1-429-444.

Khilare V C, Ahmed R, Chavan S S and Kohire O D 2009. Management of Fusarium oxysporum f. sp. ciceri by different fungicides. Bioinfolet 6: 4143.

Kohire O D, Shrirame M D, Dahiwal A L, Shirurkar P D and Vishnupurikar R M 2006. Status of chickpea wilt, dry root rot and stunt in major chickpea growing districts of Marathwada region of Maharashtra. Paper presented in Agro research conservation and management held at Raipur, on 14-15 Dec. 338 pp.

Korde M G 2011. Studies on Fusarium wilt of chickpea caused by Fusarium oxysporum f. sp. ciceri (Padwik) Synder and Hansan. M. Sc. (Agri.) thesis submitted to VNMKV, Parbhani (India).

Kumar A, Lal H C and Akhtar J 2012. Morphological and pathogenic characterization of Fusarium oxysporum f. sp. ciceri causing wilt of chickpea. Indian Phytopath. 65 (1): 64-66.

Mandhare V K, Deshmukh G P, Patil J V, Kale A A and Chavan U D 2011. 
Morphological, pathogenic and molecular characterization of Fusarium oxysporum f. sp. ciceri isolates from Maharashtra, India. Indonesian J. Agricultural Sci., 12 (2): 47-56.

Mulik M B 2009. Studies on wilt of chickpea incited by Fusarium oxysporum f. sp. ciceri (Padwick) Synder and Hansan. M. Sc. (Agri.) thesis submitted to VNMKV, Parbhani (India).

Nema K G and Khare M N 1973. A conspectus of wilt of Bengal gram in Madhya pradesh. Symposium on wilt problem and breeding for wilt resistance in Bengal gram, Sept. 1973 at Indian Agril. Res. Institute, New Delhi, India. 1-4 pp.

Nene Y L and Reddy M V 1987. Chickpea diseases and their control. Phytopathology 42 : 499-505.

Patil V B 2010. Studies on survey and management of chickpea wilt in Marathwada region. Ph. D. (Agri.) thesis submitted to VNMKV, Parbhani (India).
Pawar K B, Bendre N J, Deshmukh R B and Bhor S B 1993. Field reaction of some chickpea lines to Fusarium wilt. $J$. Maharashtra Agril. Univ. 18: 327328.

Singh K B and Dahiya B S 1973. Breeding of wilt resistance in chickpea. Symposium on wilt problem and breeding for wilt resistance in Bengal gram. September 1973 at IARI, New Delhi, India, 13-14. pp.

Suryawanshi A V, Mandhare V K, Sanap M $M$ and Jamadagni B M 2003. Reaction of chickpea entries to Fusarium wilt and gram pod borer. J. Maharashtra Agric. Univ. 28 (2): 213-214.

Trivedi S and Gurha S N 2007. Variability in Fusarium oxysporum f. sp. ciceri isolates from Jhansi district of Bundelkhand, Uttar Pradesh. J. Mycol. Pl. Pathol. 37 (2): 324-326.

Zote K K, Pawar N B, Zindurde D D, Dandnaik B P and Shirshikar S P 1993. Source of resistance to Fusarium wilt of chickpea. International Chickpea Newsletter. 29: 11-12.

\section{How to cite this article:}

Thaware, D.S., V.M. Gholve and Ghante, P.H. 2017. Screening of Chickpea Varieties, Cultivars and Genotypes against Fusarium oxysporum f. sp. ciceri. Int.J.Curr.Microbiol.App.Sci. 6(1): 896-904. doi: http://dx.doi.org/10.20546/ijcmas.2017.601.105 